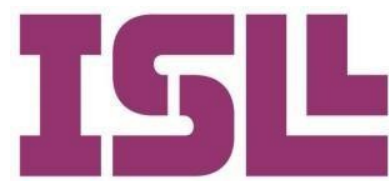

Número 11

Junio de 2019

\title{
Mejora de la comprensión lectora mediante la formulación de preguntas tipo test
}

\author{
Raúl Gutiérrez-Fresneda \\ Universidad de Alicante \\ https://orcid.org/0000-0002-3432-1676 \\ María Teresa del Olmo Ibáñez \\ Universidad de Alicante \\ https://orcid.org/0000-0001-8326-1879
}

Recibido: 03-11-2018

Aceptado: 03-04-2019

Pág. 93 a la 104

Palabras clave: lectura, aprendizaje, comprensión lectora, formulación de preguntas.

\section{Resumen:}

La lectura no es únicamente un medio de una serie de cuestiones con diferentes de acceso al conocimiento, sino que alternativas de respuesta se mejora la constituye una poderosa habilidad que capacidad comprensiva y se fomenta la permite aprender y pensar. Leer no integración de la información en los siempre conduce al aprendizaje conocimientos de los estudiantes. En el profundo, ese potencial sólo se logra trabajo participaron 118 alumnos con cuando el lector participa en edades comprendidas entre los 8 y los 9 situaciones que le exigen ir más allá del años. Los resultados otorgan valor texto. La tipología de recursos que se didáctico a este tipo de prácticas lectoras emplean en el aula para instruir en la en cuanto que facilitan la mejora de la capacidad comprensiva suele estar comprensión lectora y del propio centrada en la respuesta por parte del aprendizaje en mayor medida que otro tipo alumnado a una serie de cuestiones de situaciones de lectura.

dadas, en muchas ocasiones de carácter literal, siendo escasos los trabajos que se han efectuado respecto al modo en que el estudiante integra la información que lee mediante situaciones que le demandan un aprendizaje activo y estimulan su capacidad de pensar. El propósito de este estudio se centra en analizar si mediante dinámicas centradas en potenciar la reflexión sobre el texto a través de la formulación 


\section{Introducción}

El aprendizaje de la lectura no es solo un medio para acceder al conocimiento, sino que es una habilidad que permite desarrollar la capacidad de aprender y pensar (Norris y Phillips, 2009; Solé, 2012). Ahora bien, el proceso lector no conduce directamente a pensar o generar conocimiento, ese potencial solo se logra cuando el lector participa en situaciones que le exigen ir más allá del texto y adentrarse en su análisis e interpretación. La estrategia centrada en la formulación de preguntas demanda una serie de conocimientos y un nivel de reflexión sobre el propio proceso lector que requiere del dominio de una serie de habilidades y una elevada complejidad cognitiva. Esta tarea a su vez implica un dominio de los componentes automáticos que intervienen en la comprensión, ya que si el lector muestra dificultades en los niveles superficiales no podrá disponer de la competencia para efectuar buenas preguntas, lo que significa que cuando los recursos cognitivos del lector se orientan a resolver tareas básicas como la decodificación lectora, difícilmente podrán dedicarse a otras demandas de mayor complejidad.

Se sabe que los estudiantes que muestran buenas habilidades de lectura durante los primeros años de su escolaridad no están necesariamente capacitados para aprender a partir de la lectura, especialmente cuando se trata de textos expositivos, del libro de texto o de otros textos disciplinares (Solé, 2018). Esto se debe a que existe una serie de habilidades básicas entre las que se encuentra la decodificación, el conocimiento de aspectos convencionales, el reconocimiento de palabras habituales, la realización de predicciones en textos narrativos o textos expositivos simples que deben dominarse antes de afrontar otras destrezas de carácter más complejo. Esto se evidencia a tenor de los datos del último informe PISA publicado (OCDE, 2015), cerca del $20 \%$ de los participantes no pudo acreditar el dominio de las competencias lectoras básicas, otro $23 \%$ mostró competencias bastante limitadas en la realización de tareas simples de lectura en textos no demasiado complejos, mientras que únicamente el 6,5\% de los participantes se situó en los niveles más altos, que capacitan para una lectura más compleja, lo que indica que se precisa de algo más para mejorar la comprensión textual (Gutiérrez, 2016; Silvestri, 2006).

Las investigaciones efectuadas sobre la enseñanza de la comprensión lectora, así como aquellas que analizan las estrategias que se utilizan en situaciones didácticas señalan que los docentes utilizan como recurso didáctico de manera frecuente y natural la interrogación (Esteban, 2017; Solé, 2018; Tough, 1989). Normalmente, mediante la formulación de preguntas al final del texto, bien para comprobar lo que se ha entendido, para ayudar a recapitular lo leído, o bien para guiar la atención hacia determinados aspectos de la información escrita. También es frecuente encontrar en los libros de texto preguntas después de las lecturas con la finalidad de ayudar a los estudiantes a recordar determinadas informaciones. Ahora bien, las cuestiones que se plantean respecto a los textos en el ámbito escolar no son similares, ni presentan el mismo grado de complejidad, aunque existe acuerdo en la diferenciación de tres grandes tipos de cuestiones (Goldman y Duran, 1998; Raphael y Au, 2005; Rouet, 2006). Las denominadas preguntas literales que están dirigidas a identificar datos o a localizar información explícita en fragmentos 
del texto y están asociada a la comprensión de carácter más superficial, las preguntas inferenciales requieren integrar la información y elaborar una interpretación del texto y están asociadas a una comprensión más profunda, y las preguntas críticas que conducen a valorar la información y analizar de manera personal el texto. Los tres tipos de preguntas son relevantes en la comprensión textual para el aprendizaje y para incrementar el conocimiento.

La habilidad para formular preguntas es un componente fundamental para aprender a comprender un texto (Hoyos y Gallego, 2017), faceta en la que intervienen no solo los niveles superficiales como el procesamiento léxico, el sintáctico o la construcción de representación de la información explícita sino los niveles más profundos de comprensión, lo que implica un verdadero aprendizaje a partir de los textos, lo que permite incrementar los conocimientos del lector (Kintsch, 1998; Solé, 2018). Ahora bien, no todas las preguntas son igual de importantes, ni contribuyen de igual modo al aprendizaje, por lo que resulta relevante conocer si mediante el desarrollo de determinadas propuestas didácticas se puede mejorar la capacidad comprensiva.

La comprensión lectora es un proceso de gran complejidad en el que el lector participa de manera activa poniendo en juego una serie de estrategias y conocimientos que le permiten interaccionar con los significados del texto, creando un modelo mental a través de un proceso de construcción de hipótesis e integración de proposiciones (Calero, 2011), lo que de hacerse a través de la formulación de preguntas entre varias personas verbalizando y poniendo en común sus estrategias de lectura puede ser un recurso que puede contribuir en gran medida al logro de los mecanismos necesarios para que el aprendizaje lector sea más eficaz.

Con este propósito se efectúa el presente estudio que tiene como finalidad conocer si mediante el desarrollo de la habilidad de formular preguntas atendiendo a su tipología se mejora el aprendizaje y la capacidad comprensiva. Para ello, se compara el grado de comprensión de la lectura en dos muestras de alumnos de entre 8 y 9 años, uno que recibe intervención en el desarrollo de la habilidad para formular preguntas de diferente tipología textual y otro que sigue el programa de trabajo tradicional consistente en la lectura y en la posterior contestación a una serie de preguntas sobre la información del texto. La hipótesis que se plantea es que el alumnado perteneciente al grupo que recibe instrucción sobre las habilidades orientadas a la formulación de diferentes tipos de preguntas obtendrá un mejor rendimiento en el proceso comprensivo de la lectura.

\section{Método}

\section{Participantes}

El estudio cuenta con la participación de 118 estudiantes con edades comprendidas entre los 8 y los 9 años $(M=8.36 ; D T=0.37)$, de los cuales el $49.2 \%$ eran niños y el 50.8 $\%$ niñas. El análisis de contingencia (chi cuadrado de Pearson) entre condición y sexo no evidencia diferencias estadísticamente significativas $\left(\chi^{2}=0.58, p>.05\right)$. Todos los participantes comparten la característica de estar ubicados en un contexto sociocultural de nivel medio. 


\section{Diseño y procedimiento}

Con la finalidad de analizar el efecto que la intervención de un programa centrado en el desarrollo de las estrategias de compresión basado en la relevancia que la formulación de preguntas presenta en la mejora de la comprensión lectora, se compara el grado de adquisición de la comprensión de la información escrita en dos muestras de estudiantes de entre 8 y 9 años, uno que recibe intervención en el proceso de comprensión lectora mediante la instrucción en estrategias para la formulación de diferentes tipos de preguntas literales, inferenciales, reorganizativas y críticas) y otro que sigue el programa de enseñanza de la lectura mediante la contestación a una serie de cuestiones que se presentan en los libros de texto a través de la clase magistral tradicional. Nuestra hipótesis es que el alumnado perteneciente al programa de intervención en los que sus integrantes verbalizan y ponen en común las estrategias que se establecen a través de un texto para la formulación de sus propias cuestiones, obtendrá un mejor rendimiento en el desarrollo de la comprensión lectora.

El diseño es cuasiexperimental de medidas repetidas pretest-postest con grupo control (Campbell y Stanley, 2005). Antes y después de implementar el programa de intervención se aplicó una batería de dos instrumentos de evaluación a todos los participantes experimentales y control con la finalidad de medir la variable dependiente (aprendizaje de la comprensión lectora).

La evaluación inicial del alumnado se efectuó de forma colectiva en el aula ordinaria en el mes de enero y dentro del horario escolar. Posteriormente se implementó el programa de intervención (3 sesiones de 50 minutos semanalmente), los estudiantes experimentales estaban distribuidos en equipos interactivos y los del grupo control de manera individual según la estructura del aula tradicional. En el mes de marzo, momento en el que ya se había aplicado por completo el programa, se volvió a realizar la evaluación a todo el alumnado con los mismos instrumentos. El estudio respetó los valores éticos requeridos en la investigación con seres humanos (consentimiento informado, derecho a la información, protección de datos personales, garantías de confidencialidad, no discriminación, gratuidad y posibilidad de abandonar el programa en cualquiera de sus fases).

\section{Instrumentos de evaluación}

- Test ACL-4 (Catalá, Catalá, Molina y Monclús, 2001). Se compone una serie de textos de diferente tipología textual: narrativa, expositiva y retórica. Valora la comprensión lectora mediante la respuesta a preguntas de carácter literal, inferencial, de reorganización de la información y de valoración crítica. En cada una de las cuestiones que se plantean se debe elegir la opción correcta entre cinco alternativas. Se trata de textos cortos, pero con una estructura interna que permite inferir, jerarquizar, organizar la información y establecer relaciones entre las frases. Se concede un punto por cada respuesta correcta. La prueba presenta un coeficiente de fiabilidad de Cronbach .80.

- Evaluación de los procesos de lectura. Para la evaluación de la lectura se han utilizado dos subtest del test PROLEC-R (Cuetos, Rodríguez, Ruano y Arribas, 2007), concretamente se emplearon las pruebas de estructuras 
gramaticales y comprensión de oraciones, que están orientadas a valorar los procesos semánticos. La puntuación total en cada una de estas pruebas se obtiene asignando un punto a cada respuesta correcta. Esta prueba presenta un coeficiente de fiabilidad de Cronbach de .79.

- Escala de conciencia lectora (ESCOLA) (Puente, Jiménez y Alvarado, 2009). Es un cuestionario para la evaluación de las habilidades metacognitivas relacionadas con la lectura. Los ítems evalúan: planificación lectora (recursos para la búsqueda de información, actitud, selección de estrategias lectoras), supervisión (nivel de ajuste entre la atención y el esfuerzo que debe realizarse, uso de estrategias para la selección de la información relevante del texto, nivel de autoeficacia en el conocimiento de las herramientas lectoras), y evaluación (control del rendimiento lector, verificación de la idoneidad de las estrategias utilizadas, reconocimiento de los resultados obtenidos). Está destinada para estudiantes de entre 8 y 13 años. Se compone de 56 ítems, pero también puede ser aplicada en dos versiones reducidas de 28 ítems cada una (ESCOLA 28-A y ESCOLA 28-B). Cada ítem tiene tres opciones de respuesta que se puntúan con 0,1 y 2 . La prueba tiene un coeficiente de fiabilidad de Cronbach de .95 .

\section{Programa de intervención}

El programa para el aprendizaje de la comprensión lectora que se utilizó se compone de textos de tipología narrativa debido a que son los textos más utilizados en estas edades y se estructura en 15 sesiones de 50 minutos de duración. Tenía como objetivo desarrollar de manera explícita las estrategias cognitivas que permiten al lector construir el significado del texto a partir de los conocimientos previos, así como adquirir las destrezas necesarias para regular y controlar todo el proceso de comprensión. Con tal finalidad se instruyó a los participantes del grupo experimental en la formulación de cuestiones de diferente tipología: literal, inferencial y crítica mediante una serie de preguntas que guiaban el proceso de análisis y reflexión de los estudiantes. Estas preguntas tenían formato tipo test con varias opciones de respuesta que se elaboraban, tanto de manera individual como grupal, y eran posteriormente intercambiadas entre los compañeros quienes las respondían y después corregían mutuamente, finalizándose con una puesta en común guiada por el profesor.

Para la formulación de preguntas literales se atendió al reconocimiento de la información explícita de los textos mediante situaciones orientadas a identificar detalles, secuenciar los sucesos y hechos de manera cronológica, captar el significado de palabras y oraciones y recordar detalles y pasajes del contenido. Las pistas que se ofrecían para su formulación eran las siguientes: ¿qué?, ¿dónde?, ¿cómo?, ¿cuándo?, ¿para qué? y ¿con quién?

Las preguntas inferenciales estaban orientadas a efectuar relaciones entre partes del texto para interpretar y realizar deducciones a partir de la información que aparece de manera explícita. Para su enseñanza se atendió a predecir información, deducir mensajes, inferir el significado de palabras por su contexto, elaborar pequeños resúmenes y proponer títulos a pequeños textos. Las cuestiones que se empleaban para su ejercitación eran las 
siguientes: ¿por qué?, ¿qué pasaría antes de...?, ¿a qué se refiere cuando...?, ¿qué título pondrías?, ¿cuál es?, ¿qué significa? y ¿qué relación hay...?

Las preguntas críticas estaban orientadas a la realización de juicios de valor, análisis crítico, realización de argumentos para sostener opiniones, a distinguir un hecho de una opinión, analizar la intención del autor y establecer conclusiones del contenido, todo lo cual generaba una interacción dialógica entre los distintos compañeros del grupo que conducía a la reflexión mutua y al aprendizaje compartido. Entre las cuestiones que se utilizaban para su instrucción destacan: ¿qué opinas...?, ¿qué crees...?, ¿cómo debería ser...?, ¿qué te parece...?, ¿qué piensas de...? y ¿qué hubieras hecho...?

Por su parte el grupo control realizaba las lecturas de manera individual y colectiva para posteriormente contestar a las cuestiones de manera individual y grupal, finalizándose con la corrección conjunta de todo el grupo clase guiada por el docente.

\section{Resultados}

Con el propósito de analizar el cambio en las variables objeto de estudio se realizaron análisis descriptivos con las puntuaciones obtenidas en los test administrados en la fase pretest, postest y en la diferencia postest-pretest, así como análisis de varianza con las puntuaciones pretest (MANOVAs, ANOVAs) y análisis de covarianza (MANCOVAs, ANCOVAs) de las diferencias postest-pretest en los grupos experimental y control en las variables medidas antes y después de la intervención. En el análisis de los datos se utilizó el programa estadístico SPSS 20. Se aplicaron las pruebas de KolmogorovSmirnov y de Levene para comprobar la normalidad y la homocedasticidad de la muestra. El nivel de significación se fijó en $p<.05$. Se calcularon las medias y desviaciones estándar de las variables y para estudiar las comparaciones entre grupos se realizaron análisis multivariados de la varianza (MANOVA), para delimitar entre qué grupos se producen diferencias significativas. Además, se calculó el tamaño del efecto (d de Cohen) (pequeño $<.50$; moderado entre .50 y .79 ; grande $\geq .80$ ). Los resultados del MANOVA pretest para el conjunto de variables puso de relieve que antes de la intervención no existían diferencias significativas entre los grupos experimental y control, $F(1,52)=1.73$, $p>.05$. Sin embargo, los resultados del MANCOVA de las diferencias postest-pretest, usando las puntuaciones pretest como covariables fueron significativos $F(1,52)=2.38, p<$ .05. Estos datos ponen de manifiesto que el programa de intervención tuvo un efecto significativo. Para analizar el cambio en cada variable se realizaron análisis descriptivos y de varianza que se presentan en la Tabla 1.

\section{Cambios en la comprensión a nivel sintáctico}

Con el objeto de analizar la eficacia del programa en el desarrollo de la comprensión lectora en la eficacia decodificadora, se estudiaron los cambios en las puntuaciones obtenidas en el Test PROLEC-R. El MANOVA pretest no evidenció diferencias significativas entre experimentales y control, $F(1.52)=2.34, p>.05$, sin embargo, los resultados del MANCOVA postest-pretest, $F(1.52)=1.56, p<.05$, confirmaron diferencias significativas entre ambas condiciones. Respecto al análisis de cada variable de forma independiente en las estructuras gramaticales se observó un 
aumento mayor en los experimentales $(M=.96)$ que en el grupo control $(M=.39)$. Los resultados del ANOVA pretest pusieron de manifiesto que en esta fase no había diferencias significativas entre experimentales y control, $F(1.52)=7.42, p>.05$. Sin embargo, el ANCOVA postest-pretest evidenció diferencias estadísticamente significativas entre condiciones, $F(1.52)=12.56, p<.001$. El tamaño del efecto fue moderado $(r=.63)$. En la variable comprensión de oraciones se constatan de igual modo incrementos superiores en los experimentales $(M=1.20)$ frente a los del grupo control $(M$ $=.51)$. Los resultados del ANOVA pretest evidenciaron que a priori no existían diferencias significativas entre ambas condiciones, $F(1.52)=8.64, p<.05$, realizándose un ANCOVA de las diferencias postest-pretest que indicó diferencias significativas, $F(1.152)=15.64, p$ $<001$, siendo el tamaño del efecto grande $(r=.76)$. Lo que pone de relieve una mejora de la capacidad para reconocer la información escrita a nivel sintáctico atribuible al programa de intervención.

\section{Cambios en la comprensión semántica relacional}

Con el propósito de evaluar el impacto del programa en la comprensión semántica relacional en pequeños textos, se analizaron los cambios en las puntuaciones obtenidas en el Test ACL-4. El MANOVA pretest realizado con el conjunto de las cuatro variables medidas (comprensión literal, inferencial, reorganizativa y critica) no evidenció diferencias significativas en la fase pretest entre experimentales y control, $F(1.152)=2.43$, $p>.05$. Sin embargo, se encontraron diferencias significativas en el MANOVA postestpretest, $F(1.152)=3.61, p<.01$, al igual que en el MANCOVA postest-pretest, $\mathrm{F}(1.152)=4.21, p<.01$. Como se puede observar en la Tabla 1, en la variable comprensión literal, la muestra experimental obtiene un incremento $(M=.48)$, superior al conseguido por el grupo control $(M=.22)$. Los resultados del ANOVA pretest pusieron de manifiesto que en esta fase no había diferencias significativas entre experimentales y control, $F(1.152)=.231, p>.05$. Sin embargo, los datos del ANCOVA de las diferencias postestpretest evidenció resultados significativos, $F(1.152)=2.40, p<.05$. El tamaño del efecto fue pequeño $(r=.26)$. En la variable comprensión inferencial, la muestra experimental obtiene un incremento $(M=.68)$, superior al conseguido por el grupo control $(M=.13)$. Los resultados del ANOVA pretest pusieron de manifiesto que en esta fase no había diferencias significativas entre experimentales y control, $F(1.152)=.325, p>.05$. Sin embargo, los datos del ANCOVA de las diferencias postest-pretest evidenció resultados significativos, $F(1.152)=7.43, p<.001$. El tamaño del efecto fue moderado $(r=.63)$. En la variable comprensión reorganizativa se constatan de igual modo incrementos superiores en los experimentales $(M=.64)$ frente a los del grupo control $(M=.19)$. Los resultados del ANOVA pretest evidenciaron que a priori no existían diferencias significativas entre ambas condiciones, $F(1.152)=.439, p<.05$, realizándose un ANCOVA de las diferencias postest-pretest que indicó diferencias significativas, $F(1,152)=9.52, p<$ 001 , siendo el tamaño del efecto moderado $(r=.67)$. Al igual que en las variables anteriores, en el caso de la comprensión crítica también el grupo experimental supera en su diferencia de medias postest-pretest $(M=.61)$ a los sujetos del grupo control $(M=.18)$. El ANOVA pretest mostró que antes de empezar la intervención no había diferencias significativas entre experimentales y control $F(1.152)=.286, p>.01$, efectuándose un ANCOVA de las diferencias postest-pretest que también indicó diferencias significativas, $\mathrm{F}(1.152)=8.73, p<.001$. El tamaño del efecto fue moderado $(r=.62)$. Estos datos ponen 
de relieve una importante mejora del desarrollo de la comprensión semántica relacional en estructuras textuales atribuible al programa de intervención, que se evidencia en el incremento significativo de la comprensión literal, inferencial, reorganizativa y crítica.

\section{Cambios en las habilidades metacognitivas de la lectura}

Para evaluar si el programa fue eficaz en el desarrollo de las habilidades metacognitivas que intervienen en el aprendizaje de la lectura se analizaron los cambios en las puntuaciones logradas en la Escala de conciencia lectora (ESCOLA). El MANOVA pretest realizado para el conjunto de las variables de la prueba puso de manifiesto que no existían diferencias significativas en la fase pretest entre experimentales y control, $F(1.152)=3.15, p>.05$. Sin embargo, se encontraron diferencias significativas en el MANOVA postest-pretest, $F(1.152)=2.37, p<.01$, al igual que en el MANCOVA postestpretest, $\mathrm{F}(1,152)=3.28, p<.01$. Como se puede observar en la Tabla 1, en la variable planificación el grupo experimental obtiene una mejora $(M=.55)$, mayor que la conseguida por el grupo control $(M=.15)$. Los resultados del ANOVA pretest pusieron de manifiesto que en esta fase no había diferencias significativas entre experimentales y control, $F(1,152)=.308, p>.05$. Sin embargo, los datos del ANCOVA de las diferencias postest-pretest indicaron resultados significativos, $F(1,152)=5.06, p<.01$. El tamaño del efecto fue moderado $(r=.53)$. En la variable supervisión se constatan de igual modo incrementos superiores en los experimentales $(M=.67)$ frente a los del grupo control $(M=.24)$. En el ANOVA pretest los datos obtenidos pusieron de manifiesto que en esta fase no había diferencias significativas entre experimentales y control, $F(1.152)=.145, p$ $>$.05. No obstante, los datos del ANCOVA de las diferencias postest-pretest indicaron resultados significativos, $F(1.152)=7.26, p<.01$, siendo el tamaño del efecto moderado $(r$.65). También se produjo una mejora tendencialmente significativa en evaluación, con un aumento mayor en los experimentales $(M=.70)$ que en el alumnado perteneciente al grupo control $(M=.28)$. Los resultados del ANOVA pretest pusieron de manifiesto que en esta fase no había diferencias significativas entre experimentales y control, $F(1.152)=.243, p>.05$. Sin embargo, los datos del ANCOVA de las diferencias postestpretest indicaron resultados significativos, $F(1.152)=6.54, p<.01$. El tamaño del efecto fue moderado $(r=.62)$. Estos datos ponen de manifiesto una mejora del aprendizaje de las estrategias de lectura atribuible al programa de intervención puesto en práctica. 
Tabla 1. Medias y Desviaciones Típicas en comprensión lectora a nivel textual y resultados del análisis de varianza y covarianza para el grupo experimental y control.

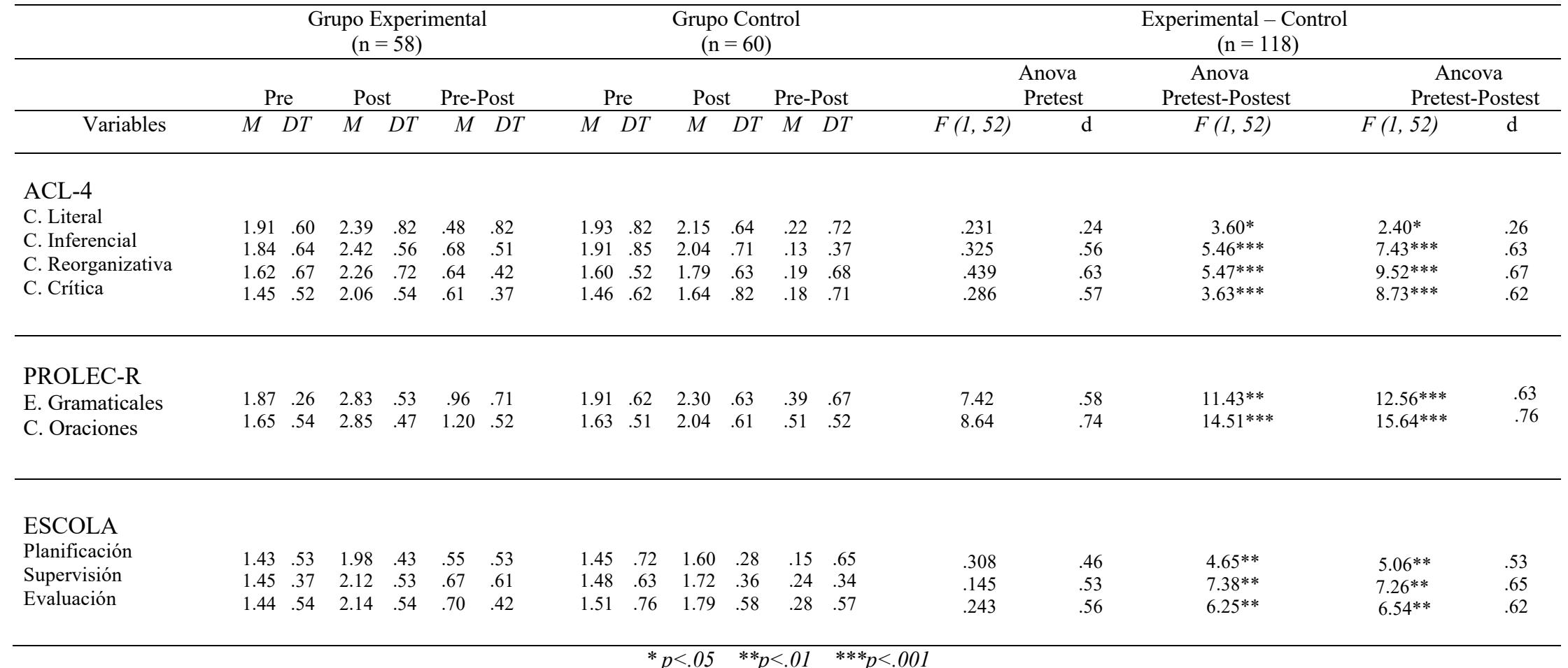




\section{Discusión y conclusiones}

El objetivo de este trabajo era comprobar si mediante la intervención de un programa orientado al desarrollo de las habilidades que contribuyen a la formulación de preguntas de diferente tipología sobre el contenido textual se producía una mejora en la capacidad comprensiva de la lectura. Los resultados obtenidos señalan que esta modalidad de enseñanza contribuye de manera significativa a la mejora de la comprensión lectora. Estos datos se encuentran en consonancia con las afirmaciones de Gutiérrez-Braojos y Salmerón (2012) quienes señalan que los estudiantes pueden mejorar el uso de estrategias si se implementan experiencias de aprendizaje adecuadas.

Uno de los motivos que pueden explicar esta mejora hace referencia a que cuando el lector busca información en el texto para formular preguntas necesita analizar e integrar la información que lee relacionando el contenido de varias secciones del texto, de modo, que es probable que la realización de ambos procesos, la generación de preguntas, junto con la búsqueda de respuestas pertinentes a las preguntas generadas por los compañeros sea un factor que contribuya a una mayor profundización en la comprensión del texto.

Se ha puesto de manifiesto en este trabajo que la comprensión lectora no surge de manera automática como producto de la decodificación, sino que es necesario además desarrollar estrategias de comprensión, resultados que son consistentes con la teoría actual. Analizando los diferentes niveles de comprensión escrita, se el grupo participante en el programa presenta un nivel superior de dominio que los alumnos del grupo control en las estructuras sintácticas, en las que el componente decodificador interviene de manera sustancial en el conocimiento de las relaciones semánticas entre las distintas partes de la oración, lo que pone de manifiesto que las estrategias lectoras influyen ya desde el nivel oracional. El acto de decodificación es una herramienta necesaria para el acceso al mundo letrado y mediante ella accedemos a descifrar el lenguaje escrito, pero ante unidades lingüísticas mayores se demanda un papel más activo del lector para poner en juego sus propios esquemas de conocimiento e interactuar con la información escrita.

Estos aportes coinciden con los postulados de Bohórquez, Cabal y Quijano (2014) al señalar que el aprendizaje de la lectura es un proceso que se produce de manera secuencial, siendo muy importante que las estrategias de comprensión se desarrollen desde los inicios del proceso lector, momento en que se adquiere el principio alfabético y los aprendices se convierten en competentes decodificadores. Respecto a la comprensión sintáctico-semántica los estudiantes pertenecientes al grupo experimental manifiestan un nivel de dominio superior, siendo estos quienes obtienen grados de comprensión superiores cuando la exigencia de la información escrita es más elevada y se demandan niveles cognitivos de mayor complejidad. Esta situación se inicia respecto a la integración de la información a nivel literal, diferencias que se incrementan cuando las demandas están orientadas a la realización de inferencias sobre la información escrita, en la reordenación de las principales ideas, así como en la capacidad para valorar el contenido textual, expresar opiniones, emitir juicios, formular preguntas al integrar la información que se va leyendo en las propias experiencias y esquemas cognitivos.

Los aportes encontrados en este trabajo coinciden con otros anteriores en los que 
se evidencia la importancia de la ejercitación en la generación de preguntas para la mejora de la comprensión lectora (Gutiérrez, 2016; Solé, 2014; Zárate, 2015). Sin embargo, en la actualidad no es esta una práctica de uso habitual en los centros escolares, por lo que a partir de los resultados de este estudio se aconseja la puesta en práctica de programas similares al realizado en este trabajo, en cuanto que existen evidencias que señalan que los estudiantes que presentan dificultades de lectura emplean escasas estrategias de comprensión, manifiestan carencias tanto en la construcción de la representación estructurada del texto, como en la realización de inferencias y en el uso del conocimiento metacognitivo (Cano, García, Justicia y García-Berbén, 2014; Ripoll y Aguado, 2014) desde los primeros niveles de la escolaridad obligatoria.

En cuanto al desarrollo de las habilidades metacognitivas de la lectura, las mejoras de los estudiantes del grupo experimental evidencian los logros en las distintas estrategias de aprendizaje del proceso lector, las cuales contribuyen a la toma de conciencia del proceso de comprensión. Esto se constata al analizar los resultados del estudio puesto que se observan progresos relevantes en las distintas estrategias que intervienen en la comprensión textual: planificación, supervisión y evaluación.

En definitiva, los progresos alcanzados por parte de los participantes del programa de intervención constatan su eficacia, lo que favorece la adquisición de las estrategias que el lector emplea para comprender un texto. Por lo que a nivel práctico se sugiere el diseño de programas orientados al desarrollo de las estrategias lectoras a partir de la instrucción en las habilidades para formular preguntas atendiendo a las diferentes tipologías textuales, tal y como se ha efectuado en este trabajo. De igual modo, sería de interés que en futuras investigaciones se considerara la influencia de estas variables en programas reeducativos orientados a la superación de las dificultades de aprendizaje de la lectura como una estrategia para la mejora de la capacidad comprensiva.

\section{Referencias}

Bohórquez, L. F., Cabal, M. A. y Quijano, M. C. (2014). La comprensión verbal y la lectura en niños con y sin retraso lector. Pensamiento Psicológico, 12(1), 169-182.

Calero, A. (2011). Cómo mejorar la comprensión lectora: Estrategias para lograr lectores competentes. Madrid: Wolters Kluwer.

Campbell, D. y Stanley, J. (2005). Diseños experimentales y cuasi experimentales en la investigación social. Buenos Aires: Amorrortu.

Cano, F.; García, A., Justicia, F. y García-Berbén, A. B. (2014). Enfoques de aprendizaje y comprensión lectora: el papel de las preguntas de los estudiantes y del conocimiento previo. Revista de Psicodidáctica, 19(2), 247-265.

Catalá, G., Catalá, M., Molina, E. y Monclús, R. (2001). Evaluación de la comprensión lectora. Barcelona: Graó.

Esteban, A. (2017). Estado actual de la comprensión lectora en Educación Primaria. Revista Fuentes, 19(1), 15-38.

Goldman, S. R. y Durán, R. P. (1988). Answering questions from oceanography texts: Learner, task and text characteristics. Discourse Processes, 11, 373-412. 
Gutiérrez, R. (2016). Efectos de la lectura dialógica en la mejora de la comprensión lectora en alumnos de Educación Primaria. Revista de Psicodidáctica, 21(2), 303-320. doi:10.1387/RevPsicodidact.15017.

Gutiérrez-Braojos, C. y Salmerón, H. (2012). Estrategias de comprensión lectora: enseñanza y evaluación en educación primaria. Profesorado: Revista de Currículum y Formación del Profesorado, 16(1), 183-202.

Hoyos, A. y Gallego, T. (2017). Desarrollo de habilidades de comprensión lectora en niños y niñas de la básica primaria. Revista Virtual Universidad Católica del Norte, $51,23-45$.

Kintsch, W. (1998). Comprehension A paradigm for cognition. Cambridge, MA Cambridge University Press.

Norris, S.P. y Phillips, L. M. (2009) Scientific Literacy. In D.R. Olson \& N. Torrance (Eds) The Cambridge Handbook of Literacy (pp. 271-285). NY: Cambridge University Press.

OECD (2015). Panorama de la educación. Indicadores de la OCDE 2015. Recuperado de: https://www.mecd.gob.es/dctm/inee/internacional/panorama-de-la-educacion2015.-informe-espanol.pdf?documentId=0901e72b81ee9fa3

Puente, A., Jiménez, V. y Alvarado, J. M. (2009). Escala de conciencia lectora (ESCOLA). Evaluación e intervención psicoeducativa de procesos y variables metacognitivas durante la lectura. Madrid: EOS.

Raphael, T. E. y Au, K. H. (2005). QAR: Enhancing comprehension and test taking across grades and content areas. The Reading Teacher, 59(3), 206-221.

Ripoll, J. C. y Aguado, G. (2014). La mejora de la comprensión lectora en español: un meta-análisis. Revista de Psicodidáctica, 19(1), 27-44.

Rouet, J. F. (2006). The skills of document use: from text comprehension to Web-based learning. Mahwah, NJ: Erlbaum.

Silvestri, A. (2006). La formulación de preguntas para la comprensión de textos: Estudio experimental. Revista Signos, 39(62), 493-510.

Solé, I. (2012). Competencia lectora y aprendizaje. Revista Iberoamericana de educación, 59, 43-61.

Solé, I. (2014) El aprendizaje de la competencia lectora. En C. Lomas (Ed.) La educación lingüística, entre el deseo y la realidad (pp. 84-95). Barcelona: Octaedro.

Solé, I. (2018). Aprender a partir de textos: preguntas para fomentar el aprendizaje. Revista Ámbitos de Psicopedagogía y Orientación, 49, 3-15.

Tough, J. (1989). Lenguaje, conversación y educación. El uso curricular del habla en la escuela desde los siete años. Madrid. Visor.

Zárate, A. (2015). El uso de las preguntas de comprensión crítica en los libros de texto. Foro de Educación, 13(19), 297-32. 


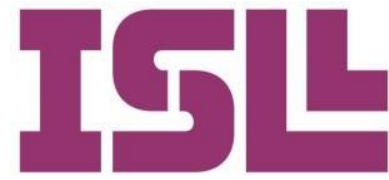

Number 11

2019 June

\section{Improved reading comprehension by formulating test questions}

Raúl Gutiérrez-Fresneda

Universidad de Alicante

https://orcid.org/0000-0002-3432-1676

María Teresa del Olmo Ibáñez

Universidad de Alicante

https://orcid.org/0000-0001-8326-1879

\section{Keywords:}

reading, learning, reading comprehension, question formulation.
Reading is not only a means of access Response alternatives, to knowledge, but it is a powerful skill comprehensive capacity is improved that allows learning and thinking. and the integration of information Reading does not always lead to deep into the student knowledge 118 learning, that potential is only achieved students with ages between 8 and 9 when the reader participates in years participated in the work. The situations that require him to go beyond results give didactic value to this the text. The typology of resources type of reading practices in that they used in the classroom to instruct in the facilitate the improvement of comprehensive capacity is usually reading comprehension and learning focused on the response by students to itself to a greater extent than other a series of questions given, in many types of reading situations.

cases of a literal nature, the work that has been done regarding the how the student integrates the information he reads through situations that demand active learning and stimulate his ability to think. The purpose of this study is to analyze whether through dynamics focused on enhancing the reflection on the text through the formulation of a series of questions with different 


\section{Introduction}

The learning of reading is not only a means to access knowledge but is a skill that develops the ability to learn and think (Norris \& Phillips, 2009; Solé, 2012). However, the reading process does not lead directly to think or generate knowledge, this potential is only achieved when the reader participates in situations that require him to go beyond the text and enter into its analysis and interpretation. The strategy focused on the formulation of questions demands a series of knowledge and a level of reflection on the reading process itself that requires the mastery of a series of skills and a high level of cognitive complexity. This task in turn implies a mastery of the automatic components that intervene in the comprehension, since if the reader shows difficulties in the superficial levels, he will not be able to have the competence to ask good questions. In other words, when the reader's cognitive resources are designated to solve basic tasks such as reader decoding, they will hardly be able to devote themselves to other more complex demands.

It is known that students who show good reading skills during the first years of their schooling are not necessarily able to learn from reading, especially when it comes to expository texts, textbooks or other disciplinary texts (Solé, 2018). This is because there are a number of basic skills including decoding, knowledge of conventional aspects, recognition of common words, making predictions in narrative texts or simple expository texts, which must be mastered before facing other skills of a more complex nature. This is evidenced by data from the latest PISA report published (OECD, 2015), about $20 \%$ of participants could not prove mastery of basic reading skills, another $23 \%$ showed fairly limited skills in performing simple reading tasks in texts not too complex, while only 6.5 $\%$ of participants were at the highest levels, which qualify for more complex reading, indicating that more is needed to improve textual understanding (Gutiérrez, 2016; Silvestri, 2006).

Research carried out on the teaching of reading comprehension, as well as that analysing the strategies used in didactic situations, indicate that teachers frequently and naturally use questioning as a didactic resource (Esteban, 2017; Solé, 2018; Tough, 1989). Normally, by formulating questions at the end of the text, either to check what has been understood, to help recapitulate what has been read, or to guide attention to certain aspects of written information. It is also common to find questions in textbooks after the readings in order to help students remember certain information. However, the questions that arise with respect to textbooks in schools are not similar, nor do they present the same degree of complexity, although there is agreement on the differentiation of three main types of questions (Goldman \& Duran, 1998; Raphael \& Au, 2005; Rouet, 2006). The so-called literal questions that are aimed at identifying data or locating explicit information in fragments of the text and are associated with understanding of a more superficial nature, inferential questions require integrating information and elaborating an interpretation of the text and are associated with a deeper understanding, and critical questions that lead to evaluate the information and analyze the text personally. All three types of questions are relevant to textual comprehension, to learning and to increasing knowledge.

The ability to formulate questions is a fundamental component for learning to understand a text (Hoyos \& Gallego, 2017), a facet in which not only superficial levels 
such as lexical processing, syntactic or the construction of representation of explicit information intervene, but also the deepest levels of comprehension, which implies a true learning from the texts, which allows increasing the reader's knowledge (Kintsch, 1998; Solé, 2018). However, not all questions are equally important, nor do they contribute in the same way to learning, so it is important to know whether through the development of certain didactic proposals it is possible to improve comprehension capacity.

Reading comprehension is a highly complex process in which the reader actively participates in an active way, putting into play a series of strategies and knowledge that allow the reader to interact with the meanings of the text, creating a mental model through a process of hypothesis construction and integration of propositions (Calero, 2011), what to do through the formulation of questions among several people verbalizing and sharing their reading strategies can be a resource that contributes greatly to the achievement of the mechanisms necessary for reader learning to be more effective.

For this purpose, the present study is being carried out in order to find out whether, through the development of the ability to formulate questions in accordance with their typology, learning and comprehension are improved. To this end, the degree of reading comprehension is compared in two samples of students between the ages of 8 and 9 , one who receives intervention in the development of the ability to formulate questions of different textual typology and the other who follows the traditional work programme consisting of reading and then answering a series of questions about the information in the text. The hypothesis is that the students belonging to the group that receives instruction on skills oriented to the formulation of different types of questions will obtain a better performance in the comprehensive process of reading.

\section{Method}

\section{Participants}

The study included 118 students between the ages of 8 and $9(M=8.36 ; T D=$ 0.37), of whom 49.2 per cent were boys and 50.8 per cent girls. Contingency analysis (Pearson chi-square) between condition and sex does not show statistically significant differences $\left(\chi^{2}=0.58, p>.05\right)$. All participants share the characteristic of being located in a socio-cultural context of medium level.

\section{Design and procedure}

In order to analyze the effect that the intervention of a program focused on the development of compression strategies based on the relevance that the formulation of questions presents in the improvement of reading comprehension, compares the degree of acquisition of the understanding of the information written in two samples of students between 8 and 9 years old, one that receives intervention in the process of reading comprehension through instruction in strategies for the formulation of different types of literal, inferential, reorganizing and critical questions) and another that follows the program of teaching reading by answering a series of questions presented in textbooks through the traditional master class. Our hypothesis is that students belonging to the intervention program in which their members verbalize and share the strategies established through a text for the formulation of their own questions, will obtain a better 
performance in the development of reading comprehension.

The design is quasi-experimental of pretest-posttest repeated measures with control group (Campbell and Stanley, 2005). Before and after implementing the intervention program, a battery of two evaluation instruments was applied to all experimental and control participants in order to measure the dependent variable (learning of reading comprehension).

The initial evaluation of the students was carried out collectively in the ordinary classroom in the month of January and during school hours. Subsequently the intervention program was implemented ( 3 sessions of 50 minutes weekly), the experimental students were distributed in interactive teams and those of the control group individually according to the structure of the traditional classroom. In March, at which time the program had already been fully implemented, the evaluation was carried out again for all students with the same instruments. The study respected the ethical values required in research with human beings (informed consent, right to information, protection of personal data, guarantees of confidentiality, non-discrimination, gratuity and the possibility of leaving the program in any of its phases).

\section{Evaluation instruments}

- ACL-4 test (Catalá, Catalá, Molina \& Monclús, 2001). It is composed of a series of texts of different textual typology: narrative, expository and rhetoric. It values reading comprehension by answering literal, inferential, information reorganization and critical appraisal questions. For each of the issues raised, the right choice must be made between five alternatives. These are short texts, but with an internal structure that allows inferring, hierarchizing, organizing information and establishing relationships between phrases. One point is awarded for each correct answer. The test has a Cronbach .80 reliability coefficient.

- Evaluation of reading processes. Two subtests of the PROLEC-R test (Cuetos, Rodríguez, Ruano \& Arribas, 2007) were used to evaluate reading. Specifically, grammatical structures and sentence comprehension tests were used to evaluate semantic processes. The total score in each of these tests is obtained by assigning one point to each correct answer. This test presents a Cronbach reliability coefficient of 0.79 .

- Escala de conciencia lectora (ESCOLA) (Puente, Jiménez \& Alvarado, 2009). It is a questionnaire for the evaluation of metacognitive skills related to reading. The items evaluate: reading planning (resources for the search of information, attitude, selection of reading strategies), supervision (level of adjustment between the attention and the effort to be made, use of strategies for the selection of the relevant information of the text, level of self-efficacy in the knowledge of the reading tools), and evaluation (control of the reading performance, verification of the suitability of the strategies used, recognition of the results obtained). It is intended for students between the ages of 8 and 13. It consists of 56 items but can also be applied in two reduced versions of 
28 items each (School 28-A and School 28-B). Each item has three response options that are scored with $0,1,2$. The test has a Cronbach reliability coefficient of 0.95 .

\section{Intervention programme}

The reading comprehension programme used consists of narrative-type texts structured into 15 50-minute sessions. The objective was to explicitly develop cognitive strategies that allow the reader to construct the meaning of the text from previous knowledge, as well as to acquire the necessary skills to regulate and control the entire comprehension process. To this end, the participants of the experimental group were instructed in the formulation of questions of different types: literal, inferential and critical through a series of questions that guided the process of analysis and reflection of the students. These questions had a multiple format with several answer options that were elaborated, both individually and in groups, and were later exchanged between the colleagues who answered them and then corrected each other, ending with a sharing guided by the teacher.

For the formulation of literal questions, attention was paid to the recognition of explicit information in texts through situations aimed at identifying details, sequencing events and facts in a chronological manner, capturing the meaning of words and sentences and remembering details and passages of the content. Among the clues offered for its formulation are the following: what, where, how, when, for what, with whom?

The inferential questions were oriented to make relationships between parts of the text to interpret and make deductions from the information that appears explicitly. In order to teach them, they tried to predict information, deduce messages, infer the meaning of words by their context, prepare small summaries and propose titles to small texts. The questions that were used for its exercise were the following: why, what would happen before..., what does it mean when..., what title would you put, what is it, what does it mean, what relationship is there...?

The critical questions were aimed at making value judgments, critical analysis, making arguments to sustain opinions, distinguishing a fact from an opinion, analyzing the author's intention and drawing conclusions from the content, all of which generated a dialogical interaction between the different companions of the group that led to mutual reflection and shared learning. Among the questions that were used for his instruction are: what do you think?, what do you think?, how should it be?, what do you think?, what do you think of...?, what would you have done?

On the other hand, the control group carried out the readings individually and collectively to later answer the questions individually and in groups, ending with the joint correction of the whole group class guided by the teacher.

\section{Results}

In order to analyse the change in the variables under study, descriptive analyses were carried out with the scores obtained in the tests administered in the pretest, postest and post pretest difference, as well as variance analysis with the pretest scores 
(MANOVAs, ANOVAs) and covariance analysis (MANCOVAs, ANCOVAs) of the post pretest differences in the experimental and control groups in the variables measured before and after the intervention. The statistical program SPSS was used in the analysis of the data. Kolmogorov-Smirnov and Levene tests were applied to check the normality and homocedasticity of the sample. The level of significance was set at $p<.05$. Means and standard deviations of variables were calculated and multivariate variance analyses (MANOVA) were performed to study comparisons between groups to delimit between which groups significant differences occurred. In addition, the effect size (Cohen d) was calculated (small $<.50$; moderate between .50 and .79 ; large $\geq .80$ ). The results of the MANOVA pretest for the set of variables showed that before the intervention there were no significant differences between the experimental and control groups, $F(1.52)=1.73, p$ $>.05$. However, MANCOVA results for post-pretest differences, using pretest scores as covariates were significant $F(1.52)=2.38, p<.05$. These data show that the intervention programme had a significant effect. In order to analyze the change in each variable, descriptive and variance analyses were carried out, which are presented in Table 1.

\section{Changes in comprehension at syntactic level}

In order to analyze the effectiveness of the program in the development of reading comprehension in decoding effectiveness, the changes in the scores obtained in the PROLEC-R Test were studied. MANOVA pretest did not show significant differences between experimental and control, $F(1.52)=2.34, p>.05$, however, the results of MANCOVA postest-pretest, $\mathrm{F}(1.52)=1.56, \mathrm{p}<.05$, confirmed significant differences between the two conditions. With respect to the analysis of each variable independently in the grammatical structures, a greater increase was observed in the experimental ones $(M=.96)$ than in the control group $(M=.39)$. The ANOVA pretest results showed that at this stage there were no significant differences between experimental and control, $F(1.52)=7.42, p>.05$. However, post-pretest ANCOVA showed statistically significant differences between conditions, $F(1.52)=12.56, p<.001$. The effect size was moderate $(r=.63)$. In the sentence comprehension variable, there are also higher increases in the experimental ones $(M=1.20)$ compared to those of the control group $(M=.51)$. ANOVA pretest results showed that a priori there were no significant differences between both conditions, $F(1.52)=8.64, p<.05$, performing an ANCOVA of post-test differences that indicated significant differences, $\mathrm{F}(1.152)=15.64, \mathrm{p}<001$, being the size of the large effect $(r=.76)$. This highlights an improved ability to recognise written information at syntactic level attributable to the intervention programme.

\section{Changes in relational semantic understanding}

In order to evaluate the impact of the program on relational semantic comprehension in small texts, changes in ACL-4 scores were analyzed. The MANOVA pretest carried out with the set of the four measured variables (literal, inferential, reorganizative and critical comprehension) did not show significant differences in the pretest phase between experimental and control, $F(1.152)=2.43, p>.05$. However, significant differences were found in the post-pretest MANOVA, $F(1.152)=3.61, p<.01$, as well as in the post-pretest MANCOVA, $\mathrm{F}(1.152)=4.21, \mathrm{p}<.01$. As can be seen in Table 1 , in the variable literal comprehension, the experimental sample obtains an 
increase $(M=.48)$, higher than that achieved by the control group $(M=.22)$. ANOVA pretest results showed that at this stage there were no significant differences between experimental and control, $F(1.152)=.231, p>.05$. However, ANCOVA data from postpretest differences showed significant results, $F(1,152)=2.40, p<.05$. The size of the effect was small $(r=.26)$. In the inferential comprehension variable, the experimental sample obtains an increase $(M=.68)$, higher than that achieved by the control group $(M$ $=.13)$. The results of the ANOVA pretest showed that at this stage there were no significant differences between experimental and control, $F(1.152)=.325, p>.05$. However, ANCOVA data from post-pretest differences showed significant results, $F(1.152)=7.43, p<.001$. The effect size was moderate $(r=.63)$. In the reorganizative comprehension variable, there are also higher increases in the experimental ones $(M=$ .64) compared to those of the control group $(M=.19)$. The results of the ANOVA pretest showed that a priori there were no significant differences between the two conditions, $F(1.152)=.439, p<.05$, with an ANCOVA of post-pretest differences indicating significant differences, $\mathrm{F}(1.152)=9.52, \mathrm{p}<001$, with moderate effect size $(\mathrm{r}=.67)$. As in the previous variables, in the case of critical comprehension also the experimental group overcomes in its difference of post-pretest means $(M=.61)$ to the subjects of the control group $(M=.18)$. The ANOVA pretest showed that before starting the intervention there were no significant differences between experimental and control $F(1.152)=.286, p>.01$, performing an ANCOVA of the post-pretest differences that also indicated significant differences, $\mathrm{F}(1.152)=8.73, \mathrm{p}<.001$. The effect size was moderate $(r=.62)$. These data highlight a significant improvement in the development of relational semantic understanding in textual structures attributable to the intervention program, as evidenced by the significant increase in literal, inferential, reorganizational, and critical understanding.

\section{Changes in metacognitive reading skills}

To assess whether the program was effective in developing the metacognitive skills involved in learning to read, changes in scores achieved on the Reading Consciousness Scale (ESCOLA) were analyzed. The MANOVA pretest carried out for the set of test variables showed that there were no significant differences in the pretest phase between experimental and control, $F(1.152)=3.15, p>.05$. However, significant differences were found in the post-pretest MANOVA, $F(1.152)=2.37, p<.01$, as in the post-pretest MANCOVA, $\mathrm{F}(1.152)=3.28, \mathrm{p}<.01$. As can be seen in Table 1 , in the planning variable the experimental group obtains an improvement $(\mathrm{M}=.55)$, greater than that obtained by the control group $(\mathrm{M}=.15)$. The results of the ANOVA pretest showed that at this stage there were no significant differences between experimental and control, $F(1.152)=.308$, $p>.05$. However, ANCOVA data from post-pretest differences indicated significant results, $F(1.152)=5.06, p<.01$. The effect size was moderate $(\mathrm{r}=.53)$. In the supervision variable there are also higher increases in the experimental ones $(M=.67)$ compared to those of the control group $(M=.24)$. In the ANOVA pretest the data obtained showed that at this stage there were no significant differences between experimental and control, $F(1.152)=.145, p>.05$. However, ANCOVA post-pretest differences data indicated significant results, $F(1.152)=7.26, p<.01$, with moderate effect size $(r=.65)$. There was also a significant trend improvement in evaluation, with a greater increase in the 
experimental $(M=.70)$ than in the students belonging to the control group $(M=.28)$. The results of the ANOVA pretest showed that at this stage there were no significant differences between experimental and control, $F(1,152)=.243, p>.05$. However, ANCOVA data from post-pretest differences indicated significant results, $F(1.152)=6.54$, $p<.01$. The effect size was moderate $(r=.62)$. These data show an improvement in the learning of reading strategiesattributable to the intervention programme implemented. 
Table 1. Typical Means and Deviations in textual reading comprehension and results of the analysis of variance and covariance for the experimental and control group.

\begin{tabular}{|c|c|c|c|c|c|c|c|c|c|c|c|c|c|c|c|c|c|}
\hline \multirow[b]{3}{*}{ Variables } & \multicolumn{6}{|c|}{$\begin{array}{l}\text { Experimental Group } \\
\quad(\mathrm{n}=58)\end{array}$} & \multicolumn{6}{|c|}{$\begin{array}{l}\text { Control Group } \\
(\mathrm{n}=60)\end{array}$} & \multicolumn{5}{|c|}{$\begin{array}{l}\text { Experimental - Control } \\
\qquad(\mathrm{n}=118)\end{array}$} \\
\hline & Pre & & Post & & re-Post & & Pre & & Post & & -Post & & $\begin{array}{l}\text { Anova } \\
\text { Pretest }\end{array}$ & & $\begin{array}{c}\text { Anova } \\
\text { Pre-Post }\end{array}$ & $\begin{array}{l}\text { Ancova } \\
\text { Pre-Post }\end{array}$ & \\
\hline & $M$ & $D T$ & $M$ & $D T$ & $M$ & $D T$ & $M$ & $D T$ & $M$ & $D T$ & $M$ & $D T$ & $F(1,52)$ & d & $F(1,52)$ & $F(1,52)$ & $\mathrm{d}$ \\
\hline $\begin{array}{l}\text { ACL-4 } \\
\text { C. Literal } \\
\text { C. Inferential } \\
\text { C. Reorganizing } \\
\text { C. Criticism }\end{array}$ & $\begin{array}{l}1.91 \\
1.84 \\
1.62 \\
1.45\end{array}$ & $\begin{array}{l}.60 \\
.64 \\
.67 \\
.52\end{array}$ & $\begin{array}{l}2.39 \\
2.42 \\
2.26 \\
2.06\end{array}$ & $\begin{array}{l}.82 \\
.56 \\
.72 \\
.54\end{array}$ & $\begin{array}{l}.48 \\
.68 \\
.64 \\
.61\end{array}$ & $\begin{array}{l}.82 \\
.51 \\
.42 \\
.37\end{array}$ & $\begin{array}{l}1.93 \\
1.91 \\
1.60 \\
1.46\end{array}$ & $\begin{array}{l}.82 \\
.85 \\
.52 \\
.62\end{array}$ & $\begin{array}{l}2.15 \\
2.04 \\
1.79 \\
1.64\end{array}$ & $\begin{array}{l}.64 \\
.71 \\
.63 \\
.82\end{array}$ & $\begin{array}{l}.22 \\
.13 \\
.19 \\
.18\end{array}$ & $\begin{array}{l}.72 \\
.37 \\
.68 \\
.71\end{array}$ & $\begin{array}{l}.231 \\
.325 \\
.439 \\
.286\end{array}$ & $\begin{array}{l}.24 \\
.56 \\
.63 \\
.57\end{array}$ & $\begin{array}{l}3.60^{*} \\
5.46^{* * *} \\
5.47^{* * *} \\
3.63^{* * *}\end{array}$ & $\begin{array}{l}2.40 * \\
7.43 * * * \\
9.52 * * * \\
8.73 * * *\end{array}$ & $\begin{array}{l}.26 \\
.63 \\
.67 \\
.62\end{array}$ \\
\hline $\begin{array}{l}\text { PROLEC-R } \\
\text { E. Grammars } \\
\text { C. Prayers }\end{array}$ & $\begin{array}{l}1.87 \\
1.65\end{array}$ & $\begin{array}{l}.26 \\
.54\end{array}$ & $\begin{array}{l}2.83 \\
2.85\end{array}$ & $\begin{array}{l}.53 \\
.47\end{array}$ & $\begin{array}{l}.96 \\
1.20\end{array}$ & $\begin{array}{l}.71 \\
.52\end{array}$ & $\begin{array}{l}1.91 \\
1.63\end{array}$ & $\begin{array}{l}.62 \\
.51\end{array}$ & $\begin{array}{l}2.30 \\
2.04\end{array}$ & $\begin{array}{l}.63 \\
.61\end{array}$ & $\begin{array}{l}.39 \\
.51\end{array}$ & .67 & $\begin{array}{l}7.42 \\
8.64\end{array}$ & $\begin{array}{l}.58 \\
.74\end{array}$ & $\begin{array}{l}11.43^{* *} \\
14.51 * * *\end{array}$ & $\begin{array}{l}12.56 * * * \\
15.64 * * *\end{array}$ & $\begin{array}{l}.63 \\
.76\end{array}$ \\
\hline $\begin{array}{l}\text { SCHOOL } \\
\text { Planning } \\
\text { Supervision } \\
\text { Evaluation }\end{array}$ & $\begin{array}{l}1.43 \\
1.45 \\
1.44\end{array}$ & $\begin{array}{l}.53 \\
.37 \\
.54\end{array}$ & $\begin{array}{l}1.98 \\
2.12 \\
2.14\end{array}$ & $\begin{array}{l}.43 \\
.53 \\
.54\end{array}$ & $\begin{array}{l}.55 \\
.67 \\
.70\end{array}$ & $\begin{array}{l}.53 \\
.61 \\
.42\end{array}$ & $\begin{array}{l}1.45 \\
1.48 \\
1.51\end{array}$ & $\begin{array}{l}.72 \\
.63 \\
.76\end{array}$ & $\begin{array}{l}1.60 \\
1.72 \\
1.79\end{array}$ & $\begin{array}{l}.28 \\
.36 \\
.58\end{array}$ & $\begin{array}{l}.15 \\
.24 \\
.28\end{array}$ & $\begin{array}{l}.65 \\
.34 \\
.57\end{array}$ & $\begin{array}{l}.308 \\
.145 \\
.243\end{array}$ & $\begin{array}{l}.46 \\
.53 \\
.56\end{array}$ & $\begin{array}{l}4.65^{* *} \\
7.38^{* *} \\
6.25^{* *}\end{array}$ & $\begin{array}{l}5.06^{* *} \\
7.26^{* *} \\
6.54^{* *}\end{array}$ & $\begin{array}{l}.53 \\
.65 \\
.62\end{array}$ \\
\hline
\end{tabular}




\section{Discussion and conclusions}

The objective of this work was to test whether the intervention of a program oriented to the development of the skills that contribute to the formulation of questions of different typology on the textual content produced an improvement in the comprehension capacity of reading. The results obtained indicate that this type of teaching contributes significantly to the improvement of reading comprehension. These data are in line with the statements of Gutiérrez-Braojos \& Salmerón (2012) who point out that students can improve the use of strategies if appropriate learning experiences are implemented.

One of the reasons that may explain this improvement refers to the fact that when the reader looks for information in the text to formulate questions, he needs to analyze and integrate the information that he reads relating the content of several sections of the text, in such a way that it is probable that the realization of both processes, the generation of questions, together with the search for pertinent answers to the questions generated by the companions will be a factor that contributes to a deeper understanding of the text.

It has been shown in this work that reading comprehension does not arise automatically as a product of decoding, but that it is also necessary to develop comprehension strategies, results that are consistent with current theory. Analyzing the different levels of written comprehension, the group participating in the program presents a higher level of mastery than the students of the control group in the syntactic structures, in which the decoder component intervenes in a substantial way in the knowledge of the semantic relations between the different parts of the sentence, which shows that the reading strategies already influence from the oral level. The act of decoding is a necessary tool for access to the literate world and through it we access to decipher the written language, but in the face of larger linguistic units a more active role of the reader is demanded in order to play their own schemes of knowledge and interact with written information.

These contributions coincide with the postulates of Bohórquez, Cabal \& Quijano (2014) in pointing out that learning to read is a process that occurs sequentially, and it is very important that comprehension strategies are developed from the beginning of the reading process, when the alphabetic principle is acquired and the apprentices become competent decoders.

With respect to syntactic-semantic comprehension, the students belonging to the experimental group manifest a higher level of mastery, being those who obtain higher degrees of comprehension when the demand for written information is higher and more complex cognitive levels are demanded. This situation begins with regard to the integration of information at a literal level, differences that increase when the demands are oriented to the realization of inferences on written information, in the rearrangement of the main ideas, as well as in the capacity to value the textual content, express opinions, issue judgments, formulate questions when integrating the information that is being read into one's own experiences and cognitive schemes.

The contributions found in this work coincide with other previous studies in which it is evident the importance that the exercise in the generation of questions presents for the improvement of reading comprehension (Gutiérrez, 2016; Solé, 2014; Zárate, 2015). However, at present this is not a practice commonly used in schools, so from the results 
of this study we recommend the implementation of programs similar to the one carried out in this work from the first school levels, inasmuch as there is evidence indicating that students with reading difficulties employ few strategies of comprehension, manifest deficiencies both in the construction of the structured representation of the text, as in the making of inferences and in the use of metacognitive knowledge (Cano, García, Justicia \& García-Berbén, 2014; Ripoll \& Aguado, 2014) from the first levels of compulsory schooling.

As for the development of metacognitive reading skills, the improvements of the students of the experimental group show the achievements in the different learning strategies of the reading process, which contribute to the awareness of the comprehension process. This can be seen in the analysis of the results of the study, since relevant progress is observed in the different strategies involved in textual understanding: planning, monitoring and evaluation.

In short, the progress made by the participants in the intervention programme shows its effectiveness, which favours the acquisition of the strategies that the reader uses to understand a text. Therefore, at a practical level, it is suggested to design programs oriented to the development of reading strategies based on the instruction in the skills to formulate questions according to the different textual typologies, as it has been done in this work. Similarly, it would be of interest in future research to consider the influence of these variables on re-educational programs aimed at overcoming learning difficulties in reading as a strategy for improving comprehension skills.

\section{References}

Bohórquez, L. F., Cabal, M. A., \& Quijano, M. C. (2014). Verbal comprehension and reading in children with and without reader delay. Psychological Thinking, 12(1), 169-182.

Calero, A. (2011). Improving Reading Comprehension: Strategies for Competent Readers. Madrid: Wolters Kluwer.

Campbell, D., \& Stanley, J. (2005). Diseños experimentales y cuasi experimentales en la investigación social. Buenos Aires: Amorrortu.

Cano, F.; García, A., Justicia, F \& García-Berbén, A. B. (2014). Approaches to learning and reading comprehension: the role of students' questions and prior knowledge. Revista de Psicodidáctica, 19(2), 247-265.

Catalá, G., Catalá, M., Molina, E., \& Monclús, R. (2001). Evaluation of reading comprehension. Barcelona: Graó.

Esteban, A. (2017). Current state of reading comprehension in Primary Education. Fuentes Magazine, 19(1), 15-38.

Goldman, S. R., \& Durán, R. P. (1988). Answering questions from oceanography texts: Learner, task and text characteristics. Discourse Processes, 11, 373-412.

Gutiérrez, R. (2016). Effects of dialogic reading on the improvement of reading comprehension in Primary Education students. Revista de Psicodidáctica, 21(2), 303-320. doi:10.1387/RevPsicodidact.15017.

Gutiérrez-Braojos, C., \& Salmerón, H. (2012). Reading comprehension strategies: teaching and assessment in primary education. Faculty: Journal of Curriculum and Faculty Development, 16(1), 183-202. 
Hoyos, A., \& Gallego, T. (2017). Development of reading comprehension skills in primary school children. Revista Virtual Universidad Católica del Norte, 51, 2345 .

Kintsch, W. (1998). Comprehension A paradigm for cognition. Cambridge, MA Cambridge University Press.

Norris, S.P., \& Phillips, L. M. (2009) Scientific Literacy. In D.R. Olson \& N. Torrance (Eds) The Cambridge Handbook of Literacy (pp. 271-285). NY: Cambridge University Press.

OECD (2015). Education at a glance OECD Indicators 2015. Retrieved from: https://www.mecd.gob.es/dctm/inee/internacional/panorama-de-la-educacion2015.-informe-espanol.pdf?documentId=0901e72b81ee9fa3

Puente, A., Jiménez, V., \& Alvarado, J. M. (2009). Reading Consciousness Scale (ESCOLA). Psychoeducational evaluation and intervention of processes and metacognitive variables during reading. Madrid: EOS.

Raphael, T. E., \& Au, K. H. (2005). QAR: Enhancing comprehension and test taking across grades and content areas. The Reading Teacher, 59(3), 206-221.

Ripoll, J. C. \& Aguado, G. (2014). Improving reading comprehension in Spanish: a metaanalysis. Revista de Psicodidáctica, 19(1), 27-44.

Rouet, J. F. (2006). The skills of document use: from text comprehension to Web-based learning. Mahwah, NJ: Erlbaum.

Silvestri, A. (2006). The formulation of questions for the comprehension of texts: Experimental study. Signos Magazine, 39(62), 493-510.

Solé, I. (2012). Reading and learning skills. Revista Iberoamericana de educación, 59, $43-61$.

Solé, I. (2014) Learning to read. In C. Lomas (Ed.) La educación lingüística, entre el deseo y la realidad (pp. 84-95). Barcelona: Octahedron.

Solé, I. (2018). Learning from texts: questions to encourage learning. Ámbitos de Psicopedagogía y Orientación Magazine, 49, 3-15.

Tough, J. (1989). Lenguaje, conversación y educación. El uso curricular del habla en la escuela desde los siete años. Madrid. Visor.

Zárate, A. (2015). The use of critical comprehension questions in textbooks. Education Forum, 13(19), 297-326. 